Conclusions Few studies used propensity score analysis, two used this method for evaluating interventions (safety or coaching programs) and only one considered work adaptations/rehabilitation. More widespread use of this methodology in large workers datasets might give information of efficiency of work adaptation when intervention studies are not suitable.

\section{PREDICTION OF OPTIMAL INTERVALS OF RADIOLOGICAL SURVEILLANCE FOR WORKERS AT DIFFERENT RISKS OF SILICOSIS - CHINA'S EXPERIENCE}

${ }^{1}$ Tse, ${ }^{1}$ Chen, ${ }^{2}$ Zhang, ${ }^{1} \mathrm{Yu},{ }^{1}$ Wong, ${ }^{3}$ Leung, ${ }^{4}$ Kromhout, ${ }^{4}$ Meijer, ${ }^{2}$ Chen. ${ }^{1}$ The Chinese University of Hong Kong, N. T., Hongkong; ${ }^{2}$ Huazhong University of Science and Technology, Wuhan, China; ${ }^{3}$ Pneumoconiosis Clinic, Department of Health, Hong Kong SAR, China; ${ }^{4}$ Utrecht University, Utrecht, Nederland

\subsection{6/oemed-2013-101717.178}

Objectives To determine the optimal intervals of chest radiographic surveillance for workers at different risks of silicosis.

Methods All 3492 workers who were exposed to silica dust during 1964-74 in an iron-ore of China were recruited into this historical cohort study, and followed up till 31/12/2008. We obtained worker's information on socio-demographics, smoking habits, disease history, and lifetime occupational history; these variables were used to develop a risk score system according to a prediction model. The discriminative ability of prediction model was determined by the area under the receiver operating characteristic (ROC) curve. We determined the optimal interval of radiographic surveillance for workers at different risk of silicosis according to the OSHA's precedent role (unacceptable risk: $>1$ / 1000).

Results The model with the best fit was the least absolute shrinkage and selection operator (LASSO) Cox model which showed a good discrimination with an area of 0.83 (95\% CI, 0.81-0.86) under the ROC curve. We classified workers into 3 risk groups according to the score chart, and found the observed probabilities matched well to the predictions. According to the OSHA's precedent role, we can determine that the initial interval of radiographic surveillance for workers in the low risk group (score $<25$ ) was 11 years and then a biyearly examination was recommended. The initial examination interval was 11 years and 5 years respectively for workers in the middle (score: 24-40) and high risk group (score $\geq 40$ ), and a yearly examination was recommended thereafter. For patients with silicosis, an annual radiological surveillance program was recommended regardless of the stage of pneumoconiosis.

Conclusions This study is the first to provide scientific evidence on the optimal intervals of radiographic surveillance for workers at different risk levels of silicosis, whilst cross-setting industry validation in subsequent studies may worth exploring.

Acknowledgement Pneumoconiosis Compensation Fund Board, Hong Kong.

\section{THE USE OF NON-RANDOMISED STUDIES IN SYSTEMATIC REVIEWS OF INTERVENTION EFFECTIVENESS: A CONTENT ANALYSIS OF COCHRANE SYSTEMATIC REVIEWS}

${ }^{1} \mathrm{~S}$ I ljaz, ${ }^{2} \mathrm{C}$ Mischke, ${ }^{2} \mathrm{~J}$ Ruotsalainen, ${ }^{3} \mathrm{~J}$ Verbeek, ${ }^{2} \mathrm{~A}$ Ojajaarvi, ${ }^{2} \mathrm{~K}$ Neuvonen. ${ }^{1} \mathrm{FIOH}$, Kuopio, Finland; ${ }^{2}$ Finland; ${ }^{3}$ The Finnish Institute of Occupational Health, Finland

10.1136/oemed-2013-101717.179
Objective Randomised controlled trials are the gold standard for evaluating interventions but especially in occupational health not always feasible. Therefore, non-randomised studies (NRS) are increasingly used as evidence for effectiveness of interventions also in Cochrane reviews. When and how NRS are included has not been evaluated to date. Our aim was to conduct an overview of practice to show what kinds of questions are addressed, what kind of methods are used and what reasons the review authors cite for the inclusion of NRS within the Cochrane Collaboration.

Methods We searched the Cochrane Database of Systematic Reviews (CDSR). We included all reviews that aimed to include NRS. We conducted study selection and data collection in duplicate and analysed the results with ATLAS.ti and Excel. We analysed how questions were addressed and reasons for inclusion were distributed over review groups, study participants and interventions.

Results We included 202 reviews. The earliest reviews were from the year 2000. The number of Cochrane reviews with NRS has consistently increased over the years. Most of the reviews (52\%) did not cite a reason. Where cited the most common reason for inclusion of NRS was non-feasibility of RCTs for an intervention (30\%). It was not always clear why RCTs were not feasible. The highest number of reviews with NRS (61) came from the EPOC group. The reviews mostly addressed health care providers $(28 \%)$. The most common tools for risk of bias assessment were EPOC group's criteria (28\%) followed by The Cochrane risk of bias tool $(15 \%)$. The assessment was not described in $3 \%$ of the reviews.

Conclusions Reasons for including NRS in systematic reviews vary across Cochrane review groups. Reasons for non-feasibility of RCTs should be better elaborated. Definition of study designs and risk of bias assessment of NRS needs more attention.

\section{OCCUPATIONAL EPIDEMIOLOGY: A BIBLIOMETRIC ANALYSIS BY COUNTRY AND ERA}

K M Venables. University of Oxford, Oxford, United Kingdom

\subsection{6/oemed-2013-101717.180}

Objectives Bibliographic databases allow the study of historical trends in research output

Methods Countries active in occupational epidemiology were identified using the EPICOH membership list. Seven countries had more than 5 member scientists: USA, Canada, Sweden, UK, Italy, France, and Netherlands. Populations in 2000 were obtained from the UN website. Papers were sought in PubMed using "occupation"” and "epidemiolog*" in Title/Abstract. Country was obtained from the "affiliation" field.

Results 7,433 papers were retrieved, the earliest from the UK in 1937 [1]. An initially steep increase in publishing has decelerated, numbers quadrupling from the 1970 s to 1980 s, doubling from 1980 s to 1990 s, but increasing by only $30 \%$ from 1990 s to 2000 s. The seven active countries together published $42 \%(3,095)$ of the total retrieved. No papers were retrieved from these countries before 1980, so results comparing them relate to 1980-2012. After correcting for population size, Sweden had the highest publication rate of 18.1 per million population, followed by Netherlands and Canada (7.5 and 6.7). USA, UK, France, and Italy were similar (5.2, 4.9, 4.9, and 4.6). In absolute numbers, the USA was the most prolific $(1,449)$. 